\title{
Development of statistical models for predicting muscle and mental activities during repetitive precision tasks
}

\section{Hilma Raimona Zadry, Siti Zawiah Md Dawal \& Zahari Taha}

To cite this article: Hilma Raimona Zadry, Siti Zawiah Md Dawal \& Zahari Taha (2016)

Development of statistical models for predicting muscle and mental activities during repetitive precision tasks, International Journal of Occupational Safety and Ergonomics, 22:3, 374-383, DOI: 10.1080/10803548.2016.1150094

To link to this article: http://dx.doi.org/10.1080/10803548.2016.1150094

\section{曲 Published online: 07 Apr 2016.}

Submit your article to this journal $₫$

Џll Article views: 66

Q View related articles $\asymp$

View Crossmark data $\nearrow$

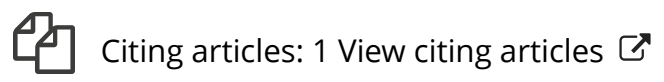




\title{
Development of statistical models for predicting muscle and mental activities during repetitive precision tasks
}

\author{
Hilma Raimona Zadry ${ }^{\mathrm{a} *}$, Siti Zawiah Md Dawal ${ }^{\mathrm{b}}$ and Zahari Taha ${ }^{\mathrm{c}}$ \\ ${ }^{a}$ University of Andalas, Indonesia; ${ }^{b}$ University of Malaya, Malaysia; ${ }^{c}$ Universiti Malaysia Pahang, Malaysia
}

\begin{abstract}
This study was conducted to develop muscle and mental activities on repetitive precision tasks. A laboratory experiment was used to address the objectives. Surface electromyography was used to measure muscle activities from eight upper limb muscles, while electroencephalography recorded mental activities from six channels. Fourteen university students participated in the study. The results show that muscle and mental activities increase for all tasks, indicating the occurrence of muscle and mental fatigue. A linear relationship between muscle activity, mental activity and time was found while subjects were performing the task. Thus, models were developed using those variables. The models were found valid after validation using other students' and workers' data. Findings from this study can contribute as a reference for future studies investigating muscle and mental activity and can be applied in industry as guidelines to manage muscle and mental fatigue, especially to manage job schedules and rotation.
\end{abstract}

Keywords: electromyography; electroencephalography; muscle activity; mental activity; precision task

\section{Introduction}

Jobs that involve constant, repetitive operations, also known as repetitive tasks,[1] performed with upper limbs and extremities are very common in industry today. Jobs are designed to be easier and repetitive so workers can carry out light tasks faster, and maximize the quality and the production outputs. Repetitive tasks involving repetitive movements of the arm comprise the majority of some tasks in the workplace, such as work in manufacturing and assembly lines.[2] Many manufacturing industries involve significant amounts of assembly work that is very hard to automate, which is why many manual jobs are still required.[3] The physical load is frequently low; however, the psycho-physiological stress levels are moderately high. Thus, assembly work is generally considered highly repetitive and standardized for extended periods of time, and may also be the main cause of musculoskeletal disorders (MSDs) in the upper extremities.[4,5]

A task is repetitive when similar exertions, actions or movements are performed often during a specific period of time. During repetitive tasks, the musculoskeletal system can begin to fatigue if enough recovery time is not provided. As the musculoskeletal system begins to fatigue, it cannot tolerate as much stress which can produce mental fatigue. Even though the amount of force applied may not change during the tasks, MSDs may occur if the musculoskeletal system is too fatigued to handle the stress.
The US Bureau of Labor Statistics in 2005 reported that repetitive motion consisting of grasping, moving and placing objects accounted for $31 \%$ of non-fatal work-related injuries in industrial workplaces in 2003.[6] A study by Dawal et al. [7] conducted in the Malaysian manufacturing and electronics industry found that the prevalence of muscle fatigue symptoms is high among industrial workers. The highest prevalence was for the neck and upper limb areas. The higher prevalence of muscle fatigue symptoms is related to job demand factors, particularly monotonous, attention requirement, precision and repetitiveness.

Worker's fatigue is one of the most prevalent issues in the workplace. It interferes with one's physical and mental operation, resulting in strength reduction and weakness. In addition, it can cause diseases, occupational accidents and a reduction in an individual's efficiency.[8] Ignoring the signs of fatigue can be dangerous to workers. Therefore, with increasing public awareness of the impact of fatigue on employee health and public safety in general, shift-work industries including mining, utility providers, medical services, transportation, on-highway transport, rail, aviation, etc., are looking to technology for solutions to this problem. Empirical studies $[9,10]$ have shown that mental stress and cognitive factors besides physical demands can produce muscle strain. Different levels of mental workload cause muscles to fatigue gradually when performing repetitive lifting tasks.[9,11] In addition, slow and fast

*Corresponding author. Email: hilma@ft.unand.ac.id 
conditions, or time pressure, may also lead to muscle fatigue.[12]

A light assembly task is a clear example of lowintensity work with elevated risks of neck and shoulder disorders.[13] Although numerous studies have investigated muscle fatigue in assembly tasks, little research has been done to explore the muscle and mental activity during a light assembly task at different precision levels.[11] Previous research on the effects of precision of a repetitive light task only measured muscle activity.[4,14-16] They did not measure mental activity during task performance. Wartenberg et al. [17] investigated the aspects of precision and speed in an assembly task (i.e., tape application). Their study, however, did not include any measures of muscle activation. Therefore, there is a need to measure fatigue taking into consideration both muscle and mental activity while performing repetitive tasks. Moreover, there are no models developed to predict both muscle and mental activities while performing repetitive precision tasks. Regarding more complex tasks (dynamic and intermittent), there have been relatively few studies; most investigate muscle fatigue during static tasks. Thus, this study aims to develop a model for predicting upper limb muscle and mental activities for different levels of repetitive precision tasks.

\section{Methodology}

\subsection{Subjects}

Fourteen subjects including seven male and seven female students from the university population were recruited to participate in the experiment. The participants' $M(S D)$ age, stature and weight were 23.15 (1.79) years, 160.01 (3.71) $\mathrm{cm}$ and 53.29 (4.40) $\mathrm{kg}$, respectively. Potential participants with any neurological or MSD history were excluded from the study. Subjects were also excluded if they had taken any medication or substance that could influence motor and neurological performance.

\subsection{Apparatus and material}

The Noraxon Telemyo 2400T Gen2 Telemetric electromyography (EMG) system complete with disposable $\mathrm{Ag} / \mathrm{AgCl} /$ solid adhesive pre-gelled surface electrodes and MyoResearch XP software version 1.07 from Noraxon USA were used to record the electrical activity of muscles. Mental activity was measured using an electroencephalography (EEG) BIOPAC MP150 system with AcqKnowledge 4.0 software and an electrode cap (CAP100C) from BIOPAC, USA. In addition, vertical electrooculograms (EOGs) were also recorded to identify blink artifacts from the recorded EEG data.

\subsection{Procedures}

In this study, the task of examining precision factor effects on muscle and mental fatigue is derived from a study by
Nakata et al.[15] This consisted of two precision levels, low precision (LP) and high precision (HP). The subjects were supplied with $14 \mathrm{~cm} \times 11 \mathrm{~cm}$ wooden trays. For the LP task, the tray had 35 holes of $6 \mathrm{~mm}$ diameter each. The HP tray had 56 holes of $1.5 \mathrm{~mm}$ diameter each. The subjects had to insert nails into the LP tray holes and colored needles into the HP tray holes, using both hands alternately. The cycle time for these tasks was measured using methods - time measurement (MTM) based on Li and Buckle's study.[18]

Each task was performed for $2 \mathrm{~h}$ in random order on two consecutive days from 9:00 until 13:00. To become familiar with the experimental equipment and procedures, a training session was performed before the experiment. The subjects were seated in a chair with the back vertical and the feet in full contact with the floor or a footrest. The desk was adjusted to elbow height so that the upper arm and forearm could form $90^{\circ}$ angles when the hand was positioned in the middle of the desk and the upper arm was vertical. Maximal voluntary contractions (MVCs) of each muscle were performed every day before the start of each experiment.

\subsection{Data collection}

\subsubsection{Maximal voluntary contraction}

MVC is the maximum amount of force that a subject will voluntarily produce and is measured at the start of each experimental day. Each MVC is performed three times, and each time is held for $5 \mathrm{~s}$ with a 30 -s rest between contractions for recovery time. The MVC refers to the highest EMG amplitude from three MVC recordings. It is applied to normalize the recording of surface EMG signals during task performance. MVC measurement procedures were carried out according to Konrad's guidelines.[19]

\subsubsection{Surface electromyography}

EMG signals were recorded from eight muscles: brachioradialis, biceps brachii, anterior deltoid and descending part of the upper trapezius, on the right and left hands. These muscles were selected because they are often involved in repetitive movements of upper extremities.[20] They are also the important muscles that elevate the arm and upwardly rotate the scapula.[21] The trapezius muscle is critical for clinical use because it reveals a high frequency of pain symptoms because of repetitive occupational tasks. Therefore, it is frequently chosen as a muscle alternative to determine electrical activity during repetitive occupational tasks.[22]

Bipolar $\mathrm{Ag} / \mathrm{AgCl}$ surface electrodes were placed at an inter-electrode distance of $20 \mathrm{~mm}$ at the belly of the muscles. A reference electrode was placed on the pisiform bone. The electrode positions were located according to 
Hermens et al.[23] They were marked with a waterproof pencil to place the electrodes at the exact same positions for both conditions. Before the electrodes were applied, the skin was cleaned with alcohol. The recording was started after the inter-electrode resistance was less than 10 $\mathrm{k} \Omega$. Data were continuously recorded using the Noraxon Telemyo 2400T Gen2 Telemetric EMGsystem.

\subsubsection{Electroencephalography}

EEG was used to record the brain activity simultaneously with the EMG during the experiments. EEG was recorded using an $\mathrm{AgCl}$ electrode cap, with electrodes placed at $\mathrm{F}$, $\mathrm{F} 4, \mathrm{Fz}, \mathrm{Pz}, \mathrm{O} 1$ and $\mathrm{O} 2$ of the International 10-20 electrodes placement system and with an electronic earlobe reference.[24] Electrode placements on F3, F4 and Fz were chosen because these locations represent the intentional and motivational centers. On the other hand, Pz points represent the activity of perception and differentiation, [25] and $\mathrm{O} 1$ and $\mathrm{O} 2$ are where the primary visual area is located. Data were continuously recorded for $2 \mathrm{~h}$ with an MP150 system and analyzed with AcqKnowledge 4.0 software from BIOPAC. Figure 1 shows the electrode placements based on the international 10-20 electrodes placement system.[24]

Electrode impedance was checked before the experiment to ensure it was $5 \mathrm{k} \Omega$ or less. The bipolar recording technique was used to record the signals. Eye movements were recorded by means of an EOG. A right-eye EOG was obtained with electrodes positioned above and below the eye with a ground on the masseter. The EOG signal was used to identify blink artifacts in the EEG data as well as changes in blink types, such as the small and slow blinks that characterize fatigue.

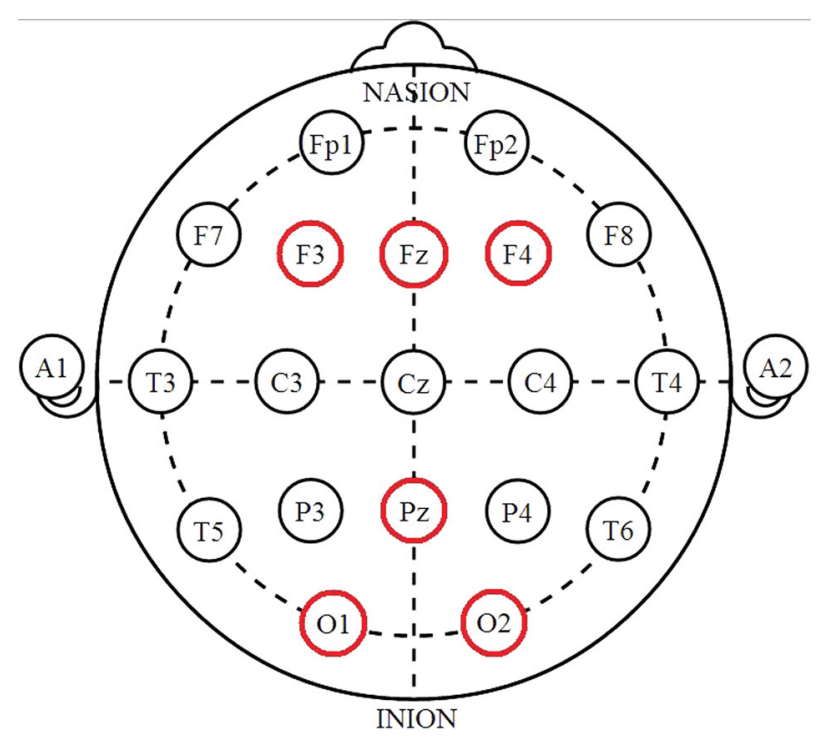

Figure 1. Placement of electroencephalography electrodes.

\subsection{Data analysis}

\subsubsection{Electromyography signal processing}

MyoResearch XP software was used to process EMG data offline. The raw EMG data were sampled during the test contraction with a sample frequency of $1500 \mathrm{~Hz}$ and band-pass filtered $(20-400 \mathrm{~Hz})$.[26] The ECG spikes due to EMG artifacts are filtered without affecting the true EMG amplitude and power spectrum. Since the 1980s, EMG has been the leading technique in assessing muscle fatigue.[27] The most popular method of analyzing EMG is monitoring changes in root mean square (rms) amplitude and mean power frequency/median power frequency (MPF/MdPF).[28] The rms amplitude of EMG provides more information than other processing methods [29] and is reported to allow for consistent, valid and accurate measurement of noisy, problematic signals.[30] Therefore, in this study, the rms values of the EMG data were analyzed. The rms values correspond to the square root of the average power of the raw EMG signal for a given period of time.[31] After calculation, the rms was normalized to the highest MVC value derived from the previous MVC test, and expressed as the maximal voluntary capacity percentage (\%MVC). The normalized rms (\%MVC) was averaged for every $5 \mathrm{~min}$, resulting in one value for every $5 \mathrm{~min}$. Consequently, 24 normalized rms values were obtained during the tasks and used in the statistical analyses. The mean values of normalized EMG rms represent muscle activities in the study. A simultaneous increase of the rms is generally considered an indication of muscle fatigue.

\subsubsection{Electroencephalography signal processing}

The signals were band-pass filtered between 1.0 and 100 $\mathrm{Hz}$ and recorded digitally $(1000 \mathrm{~Hz}$ sample frequency). The EEG was checked offline for EOG artifacts. The data were transformed to a fast Fourier transform, using a $100 \%$ Hanning window. Then, power values were generated in five separate frequency bands after averaging. This study analyzed the EEG $\alpha$ band that is defined as the frequency between 8 and $13 \mathrm{~Hz}$. Then, the mean power of the power spectrum within the epoch (mean power, in volts squared/hertz) was calculated. The mean power of the EEG $\alpha$ band was calculated for every 5 min, resulting in 24 mean $\alpha$ power readings during the tasks. These values were then normalized by dividing the mean $\alpha$ power with the initial value of each measurement. These normalized values were used in the statistical analyses. The mean values of normalized EEG $\alpha$ power represent mental activities in the study. A simultaneous increase of $\alpha$ power is generally considered an indication of mental fatigue (drowsiness).

\subsubsection{Statistical analysis}

Statistical methods using SPSS version 19.0 were used to analyze data derived from EMG and EEG software analysis. The data were tested for normality distribution 
before being used for further analysis using the ShapiroWilk test. Hence, the non-parametric statistic (Wilcoxon signed-rank test) was used to investigate the differences of muscle and mental activities between different task levels because the data were not distributed normally, where $p<0.05$ was regarded as statistically significant.

Models were developed on different levels of repetitive precision tasks using regression analysis, with the muscle activity as the dependent variable and the mental activity and time interval as the independent variables. The time interval refers to the time used to perform the task. An interval representing every $5 \mathrm{~min}$ performs the task. Thus, there are 24 time intervals obtained during $2 \mathrm{~h}$ of tasks. The models were developed with a $95 \%$ confidence level. The models were then validated using data from other students (three male and three female) and industrial workers (five male and five female).

The standard error of estimate ( $S E E$ ) measures the error or accuracy of the prediction for the validation process. The $S E E$ is an indicator of the average error of prediction for the regression equation. The better the fit of the regression line, the less variability there will be around it and the smaller the SEE.[32,33] A small SEE value is considered evidence of validation.

\section{Results and analysis}

\subsection{Muscle activity}

Table 1 presents the mean of normalized EMG rms for LP and HP tasks, as well as the significant difference values between task levels. This illustrates that muscle activity in the HP task was higher than in the LP task. However, the differences were not statistically significant $(p>0.05)$, meaning that different task levels did not affect muscle activity during task performance.

Table 1 also demonstrates that the right upper trapezius muscle has the highest muscle activity in both tasks (12.667\% and $15.786 \%$, respectively). Muscle activity of the left upper trapezius followed (10.653\% and $14.284 \%)$, while the right biceps brachii muscles have the lowest muscle activity $(5.255 \%$ and $6.036 \%)$.

\subsection{Mental activity}

The means of normalized EEG $\alpha$ power in all channels is presented in Table 2, which reveals that mental activity in the O1-O2 channels was higher than the other channels for both task levels (1.482 and 1.962, respectively). This is followed by mental activity of the $\mathrm{Fz}-\mathrm{Pz}$ and $\mathrm{F} 3-\mathrm{F} 4$ channels, indicating that tasks affect the subjects' visual activity, since the $\mathrm{O} 1-\mathrm{O} 2$ channels are located near the primary visual area in the brain.

Table 2 also presents the $p$ value of the Wilcoxon signed-rank analysis for determining differences in mental activity between task levels. This shows that mental activity for the HP task was relatively higher than for the LP task, except in the F3-F4 channels. A significant difference was only found in the $\mathrm{O} 1-\mathrm{O} 2$ channels $(p=0.011)$, indicating that different task levels affect mental activity of the $\mathrm{O} 1-\mathrm{O} 2$ channels differently, something related to visual activity.

\subsection{Development of the regression model}

Regression analyses were carried out to identify the relationships between upper limb muscles and mental fatigue over time. Analysis of muscle and mental activities showed

Table 1. Muscle activity (\%MVC) for low-precision and high-precision tasks.

\begin{tabular}{lcccc}
\hline Muscle & Mean rms of LP & Mean rms of HP & \% difference & Sig. (2-tailed) \\
\hline R. brachioradialis & 8.048 & 9.700 & 17.026 & 0.140 \\
L. brachioradialis & 6.788 & 8.155 & 16.766 & 0.594 \\
R. biceps brachii & 5.255 & 6.036 & 12.942 & 0.778 \\
L. biceps brachii & 7.072 & 9.065 & 21.987 & 0.196 \\
R. anterior deltoid & 6.964 & 6.468 & 7.681 & 0.778 \\
L. anterior deltoid & 5.883 & 12.327 & 52.281 & 0.198 \\
R. upper trapezius & 12.667 & 15.786 & 19.759 & 0.397 \\
L. upper trapezius & 10.653 & 14.284 & 25.422 & 0.245 \\
\hline
\end{tabular}

Note: $\% \mathrm{MVC}=$ maximal voluntary capacity percentage; $\mathrm{LP}=$ low precision; $\mathrm{HP}=$ high precision; sig. $=$ significance; $\mathrm{R}=$ right; $\mathrm{L}=$ left.

Table 2. Mental activity for low-precision and high-precision tasks.

\begin{tabular}{lcccc}
\hline EEG channel [24] & Mean $\alpha$ power of LP & Mean $\alpha$ power of HP & \% difference & Sig. (2-tailed) \\
\hline F3-F4 & 1.304 & 1.265 & 3.122 & 0.925 \\
Fz-Pz & 1.269 & 1.271 & 0.106 & 0.925 \\
O1-O2 & 1.482 & 1.962 & 24.491 & 0.011 \\
\hline
\end{tabular}

Note: $\mathrm{EEG}=$ electroencephalography; $\mathrm{LP}=$ low precision; HP = high precision; $\mathrm{LP}=$ low precision; sig. = significance. 
that the right upper trapezius muscle has the highest muscle activity and muscle fatigue rate compared with other muscles for both LP and HP tasks. The O1-O2 channels have the highest mental activity among all channels for LP and HP tasks.

Muscle and mental activities also have a highly significant relationship with the time interval. Therefore, to develop a regression model for muscle and mental activities, only the variables of muscle activity of the right upper trapezius, mental activity of the $\mathrm{O} 1-\mathrm{O} 2$ channels and the time interval were utilized in the linear regression analysis. The dependent variable was muscle activity of the right upper trapezius, while the independent variables were mental activity of the $\mathrm{O} 1-\mathrm{O} 2$ channels and the time interval. A regression model was developed for both LP and HP tasks, and the results are presented in Table 3.

The resulting equations are as follows:

Low-precision task: muscle activity $=9.352$

$$
+1.786 \text { (mental activity) }+0.053 \text { (time interval) }
$$

High-precision task: muscle activity $=7.907$

+5.434 (mental activity) -0.223 (time interval)

Table 3 shows that the HP task model has a very high coefficient of determination $\left(R_{\text {adj }}^{2}=0.950\right)$, suggesting that the model fits the data well. $R_{\text {adj }}^{2}$ is the proportion of variance in the dependent variable, which can be predicted from the independent variable. It signifies that $95 \%$ of the model fits the population. On the other hand, the LP task coefficient of determination can be categorized as medium $\left(R_{\text {adj }}^{2}=0.644\right)$. However, the significant model value is less than $0.05(p<0.001)$ and the $S E E$ is relatively small $(S E E=0.613)$. Therefore, the relationship is reliable and can be used to make predictions.

\subsection{Model validation}

Model validation used data from six students and 10 industrial workers. $S E E$ was used to validate the data predicted by the models. Table 4 presents the actual values $(Y)$ and the prediction values $\left(Y^{\prime}\right)$ of models as well as the models' SEE after validation.

Table 4 shows the models' actual values, prediction values and $S E E$ values after validation, and indicates that the actual and prediction values are not that different. The LP task model $S E E$ values are smaller compared with the HP task $S E E$ values. The $S E E$ values of the workers' data are higher than the $S E E$ values of the students' data. This might be due to background differences between students and workers, such as age. Workers are relatively older than students; therefore, workers' rates of muscle and mental fatigue might be higher than those of students. However, the $S E E$ values for all models are relatively small, signifying that the models are valid.

\section{Discussion}

\subsection{Muscle activity}

The highest indication of muscle activity was detected in the right upper trapezius muscle area. The reason for this issue might be that the subjects are all right handed. This could be the left upper trapezius if they are left handed. The load on the upper trapezius during the task was consistent with prior data on, for example, sewing machine operators,[34] carpenters [35] and electronics assembly workers.[7,11] These results were also consistent with other studies showing that the shoulder elevators are mainly sensitive to fatigue when performing tasks with the upper limb at or above shoulder level.[36,37]

Some studies postulated on the role of the trapezius muscle in posture. This muscle is located at the base of the neck, down towards the shoulder and arm, and passes

Table 3. Model summary of regression analysis for low-precision and high-precision tasks.

\begin{tabular}{lcccccc}
\hline & & & \multicolumn{2}{c}{ Regression coefficients } & & \\
\cline { 4 - 5 } Dependent variable & Task & Intercept & Mental activity & Time interval & \multirow{2}{*}{$R_{\text {adj }}^{2}$} & SEE \\
\hline Muscle activity & LP & 9.352 & 1.786 & 0.053 & 0.644 & 0.613 \\
& HP & 7.907 & 5.434 & -0.223 & 0.950 & 0.335 \\
\hline
\end{tabular}

$p<0.001$.

Note: $\mathrm{LP}=$ low precision; $\mathrm{HP}=$ high precision; $R_{\mathrm{adj}}^{2}=$ coefficient of determination;

$S E E=$ standard error of estimate.

Table 4. Model validation.

\begin{tabular}{|c|c|c|c|c|c|c|c|}
\hline \multirow[b]{2}{*}{ Model } & \multirow[b]{2}{*}{ Task } & \multicolumn{3}{|c|}{ Students } & \multicolumn{3}{|c|}{ Workers } \\
\hline & & Actual values $(Y)$ & Prediction values $\left(Y^{\prime}\right)$ & SEE & Actual values $(Y)$ & Prediction values $\left(Y^{\prime}\right)$ & SEE \\
\hline \multirow[t]{2}{*}{ Muscle activity } & LP & 12.926 & 12.295 & 1.331 & 12.703 & 13.707 & 2.040 \\
\hline & HP & 12.302 & 12.995 & 1.483 & 23.573 & 18.728 & 5.446 \\
\hline
\end{tabular}

Note: $\mathrm{LP}=$ low precision; $\mathrm{HP}=$ high precision; $S E E=$ standard error of estimate. 
under the shoulder blade. When this muscle shortens and tightens, it generates neck pain, usually caused by 'tension, stress, lack of exercise, poor ergonomics, or keeping a sitting posture for long time'.[38, p.136] In the case of this study, high muscle activity in the upper trapezius was also contributed by lengthy sitting postures and repetitive tasks.

Muscle activity relatively increased in the higher level task than in the lower one. These results were in line with those of a previous study by Escorpizo and Moore.[16] They found that light manual precision work increases shoulder muscle activity as revealed by EMG. This is thought to reflect a decrease in conduction muscle fiber velocity, which is related to the onset of fatigue.[39] However, significant differences in muscle activity between precision levels were not found in all muscles.

Some possible reasons for the lack of significant differences include the following. First, the effect of speed on the task is a possible explanation. A study by Szeto and Lin [14] illustrated that speed and precision demands are frequently correlated and affect the EMG. The study showed that a high-speed precision demand combination produced the main effect on EMG results. Furthermore, precision had small or no influence on EMG at low speed. In this study, the speed for LP and HP tasks is similar and determined by MTM. Therefore, precision had little influence on EMG. Secondly, similar to the reasons stated for pace tasks, methodological limitations might explain the lack of effects in this study. The object weights used in both tasks were almost the same and very light; they were, therefore, too small to be reflected by EMG.

The EMG results obtained were studied and evaluated, and the evidence suggests that muscle activity in the precision tasks were influenced not only by precision levels, but also by task speed. In spite of the varying consequences of repetitive tasks on muscle activity, it is significant to reflect on the physiological explanation following the repetitive task. Hägg [40] stated that there are motor units in the muscles that are directly affected even by gentle contractions. In addition, for each relatively large contraction, such motor units are stimulated ahead of the larger ones. These motor units can relax only after the larger muscles have wholly relaxed. More specifically, these motor units remained active during the contraction movement and following the relaxation of large muscles.[40] Unfortunately, relaxation was not complete and the motor units were continuously active in the repetitive tasks, thus contributing to pain and muscle fatigue. Waersted [41] supported Hägg's theory by stating that the constant activation of these small muscle motor units may contribute to deterioration, injury and then pain.

\subsection{Mental activity}

Similar to the results for muscle activity, the results demonstrate increasing EEG $\alpha$ power with the increase of time (duration of task). The increases occurred in all channels for all tasks. Increasing EEG $\alpha$ power indicates that the subjects were experiencing drowsiness. These results were in agreement with several EEG studies related to driving, where EEG $\alpha$ and $\theta$ power increased as the driver's level of alertness decreased.[42,43] Activity of $\alpha$ reflects a relaxed wakefulness state, and decreases with concentration, stimulation or visual fixation. Previous studies provided evidence that $\alpha$ activity increased during fatigue.[44,45]

Other EEG measurement results show that mental activity was highest in the $\mathrm{O} 1-\mathrm{O} 2$ channels for all tasks (pace, precision and load). The explanation for this might be that $\mathrm{O} 1$ and $\mathrm{O} 2$ in EEG channels are near the primary visual area.[25] The eyes were forced to work harder when performing the tasks for $2 \mathrm{~h}$ with no break. Subsequently, the subjects become drowsy, an indication of mental fatigue. The mental activity in this area was higher compared with the others. When comparing different task levels, significant difference between different mental activities was only found in the $\mathrm{O} 1-\mathrm{O} 2$ channels. This strengthens the results that especially precision tasks affect mental activity related to the visual area. This could be because more attention and alertness were needed for the precision tasks.

Analysis results show significantly high correlations between time interval, muscle activity and mental activity, indicating that muscle and mental activity increases with an increase of time. This reflects subjects experiencing physical and mental fatigue after performing the tasks. The strong relationship between muscle and mental activity can be explained by direct correlation between the electrical activity of the motor cortex and muscle fatigue. The change in the power of EEG signals is an indicator of a metabolic process in the motor cortex which sustains the MVC after muscle fatigue. Casey et al. [46] have another theory on the relationship between muscle and mental fatigue. Muscle contraction stimulus starts in the brain; therefore, muscle fatigue may also be triggered if the central nervous system cannot stimulate the neuromuscular junction. The brain's inability to stimulate the neuromuscular junction is known as central fatigue and may be caused by a lack of concentration or alertness, or loss of motivation.[46] Other studies found that the power of EEG signals also changed as muscle fatigue changed.[47]

The results which found the strong relationship between muscle activity and mental activity were consistent with some previous study results. A study by Waersted [41] evidenced that mental activity contributed to muscle fatigue especially in the neck and shoulder regions. Upper trapezius muscle activity also increased while performing the task with continuous visual attention and high levels of mental processing.[48] Besides arm posture and hand activities, some studies showed that a simultaneous mental effort also increases muscle activity ahead of the desired task alone.[9,41,49] 
Research by Hughes et al. [9] found that increasing mental workload while typing can result in increased muscle activity. During isometric work, blood flow increased in the neck-shoulder muscles upon exposure to mental stress, as did muscle tension. This would appear to be of clinical significance in work conditions which involve repetitive static load to the neck-shoulder muscles and exposure to mental stress.[50] The increase in spinal load was directly attributable to increased muscle activity.

This study has shown that not only physical demands but also cognitive factors and mental stress may induce muscle fatigue. This means that ongoing psychological stress may keep low-threshold motor units active more or less continuously. This supports some previous study findings.[10,50,51] Nevertheless, these study results show that mental load could change muscle activity when combined with physical tasks which support the previous study results. [50,52] Thus, it can be concluded from this study that muscle and mental activities both contribute to muscle and mental fatigue.

The proposed model uses linear regression analysis since a linear fit yielded the best result for the relationship between muscle activity, mental activity and time interval. Two equations for low-level and high-level tasks were developed for each repetitive task. EMG rms of the right upper trapezius was selected as a variable of interest. Then, a good linear fit was found for the $\mathrm{O} 1-\mathrm{O} 2 \alpha$ power and time interval. A high-quality relationship was found between muscle activity, mental activity and time interval. The $R_{\text {adj }}^{2}$ values were mainly above 0.8 except for the LP task $\left(R_{\text {adj }}^{2}=0.644\right)$, meaning that the established models were able to explain more than $80 \%$ of data variability.

These study results support the study by Coorevits et al. [53] in that the use of simple linear regression techniques still seems the most appropriate when studying muscle fatigue. Furthermore, according to Roman-Liu et al.,[1] regression analysis is the most reliable way of expressing changes of the parameter in time. Solnik et al. [54] also developed a statistical model using linear regression to describe the EMG signal frequency changes during submaximal isometric contraction.

The regression models show that the slopes for mental activity variables were higher for high-level tasks than low-level tasks for all tasks (e.g., $\beta_{1}=0.273$ for low load, $\beta_{1}=3.195$ for high load). This evidences that mental activity in a higher level task tends to contribute higher muscle activity and muscle fatigue. The models also show that muscle activities have high correlation with mental activities for both levels. This is because of the involvement of mental demands (e.g., attention, monotonous, time pressure/stress) in the precision tasks. These results were consistent with results from previous studies.[55] Therefore, an effort to manage muscle and mental activity through job/rest schedule, job rotation or job variation is important to reduce fatigue on workers.
The developed models were validated using data from other students and workers. Results show that the models' $S E E$ values for both tasks were relatively small, thus validating the models. The smallest value of the standard errors is zero, when all of the points fall along the equation line. There is no upper limit, so it can be hard to judge by itself. Compared with the other models, the model with the smallest $S E E$ is the best fit for the sample. However, the $S E E$ values were higher when models were validated using workers' data, potentially due to some subject related factors such as age and job tenure. Workers who participated in this study are relatively older and have higher job tenure than students. Therefore, the SEE values were higher when models were validated by workers' data rather than students' data. This result supports prior studies that have identified a positive association between age, job tenure and disorder.[56,57]

The acceptable fatigue risk is hotly debated in modelbased fatigue risk management in commercial aviation and other transportation modes.[58] A quantitative approach to addressing this issue, referred to by the Federal Aviation Administration with regard to its final rule for commercial aviation 'Flight Crew Member Duty and Rest Requirements', is to compare predictions from a mathematical fatigue model against a fatigue threshold.

Internationally, no firm recommendations exist about what is or what is not an acceptable level of physical or mental fatigue in the workplace. This is because of the complexity of the subject, the difficulty of translating laboratory measures to the everyday workplace and the specific interaction between each individual's work and the rest of their life. Shift-work is viewed by experts and by those who do it as a powerful provoker of fatigue and as a constant source of stress for workers. Human resource policies should acknowledge the various problems in this field that can arise at work. Most of all, managers should have a basic idea of how to recognize and deal with the early signs that one of the people in their care is beginning to be exposed to health and safety problems related to fatigue from whatever source.

However, this study follows the study by Byström et al.[59] The acceptable level of fatigue is defined when muscle activity derived from the developed statistical models has exceeded $25 \%$. Furthermore, the acceptable level of fatigue can also be derived by comparing the predictions from the models against a fatigue threshold study.[58] The fatigue threshold can be investigated through data from subjective measurements (questionnaire). Muscle fatigue can then be determined. Thus, scheduling options for use in industry can be generated.

Nevertheless, the development and validation of an upper limb muscle and mental fatigue model is essential if one is to predict reliably upper limb muscle activity or mental activity with a given time during repetitive tasks. The models incorporated combined upper limb muscle 
activity, mental activity and time variables which have not been done previously, while most methods developed models of muscle and mental fatigue independently.

\section{Conclusion}

This study investigated upper limb muscle and mental activities using surface EMG and EEG while performing repetitive precision tasks. It was found that repetitive tasks conducted in this study involve both upper limb muscle and mental activities and result in upper limb muscle and mental fatigue at the end of the tasks. Different levels of task contribute to different muscle and mental activities.

There is a strong linear relationship between muscle and mental activities in the majority of EMG muscles and EEG channels, indicating that if muscles were fatigued, mental fatigue also occurred. Moreover, the analysis results show significantly high correlations between the time interval, muscle activity and mental activity, indicating that muscle and mental activities increase with an increase of time. This indicates that subjects experienced both physical and mental fatigue after performing the tasks.

Regression models, utilizing muscle activity, mental activity and time variables, have been developed to predict upper limb muscle and mental activity while performing repetitive tasks. The models were valid and may be potentially used as design guidelines to manage upper limb muscle and mental fatigue for repetitive tasks in industry.

Other results from the study also show that the right upper trapezius muscle was found to be the critical muscle for muscle fatigue in all tasks. Muscle activity in this muscle was higher than in others. The rationale for this issue might be that the subjects are all right handed. The results might be different if the subjects were left handed. Thus, the prediction models may apply only to right-handed workers.

In addition, the occipital area of the brain which is near the visual region was found to be the critical zone of mental fatigue in all tasks. Therefore, the prediction models use the EMG rms of the right upper trapezius as a dependent variable, while mental activity of the $\mathrm{O} 1-\mathrm{O} 2$ channels and the time interval were independent variables.

\section{Disclosure statement}

No potential conflict of interest was reported by the authors.

\section{Funding}

The author is grateful to the eScienceFund [MOSTI No. 0301-03-SF0064] and Dana BOPTN Universitas Andalas [No. 08/UN.16/PL/AKS/2014] for supporting this research.

\section{References}

[1] Roman-Liu D, Tokarski T, Wójcik K. Quantitative assessment of upper limb muscle fatigue depending on the conditions of repetitive task load. J Electromyogr Kinesiol. 2004;14(6):671-682.

[2] Fuller JR, Lomond KV, Fung J, et al. Posture-movement changes following repetitive motion-induced shoulder muscle fatigue. J Electromyogr Kinesiol. 2009;19(6):10431052.

[3] Hilton M. Research on future skill demands: a workshop summary. national research council of the national academies. Washington (DC): National Academies Press; 2008.

[4] Nordander C, Balogh I, Mathiassen S, et al. Precision of measurements of physical workload during standardised manual handling. Part I: surface electromyography of $\mathrm{m}$. trapezius, $\mathrm{m}$. infraspinatus and the forearm extensors. J Electromyogr Kinesiol. 2004;14(4):443-454.

[5] You H, Kwon O. A survey of repetitiveness assessment methodologies for hand-intensive tasks. Int J Ind Ergon. 2005;35(4):353-360.

[6] US Bureau of Labor Statistics. Incidence rates for nonfatal occupational injuries and illnesses involving days away from work per 10,000 full-time workers by event or exposure leading to injury or illness and selected sources of injury or illness 2003. US Department of Labor; 2005.

[7] Dawal SZ, Ghazilla RAR, Zadry HR, et al. incorporating ergonomics evaluation in assembly and disassembly of repetitive task: focusing on load task. Adv Mat Res. 2013;712-715:2879-2883.

[8] Moradifar R, Hoveidi H, Givehchi S, et al. Examining fatigue and insomnia symptoms among workers of a gas transmission industry in 2013. Electronic Physician. 2014;6(2):827-31.

[9] Hughes L, Babski-Reeves K, Smith-Jackson T. Effects of psychosocial and individual factors on physiological risk factors for upper extremity musculoskeletal disorders while typing. Ergonomics. 2007;50(2):261-274.

[10] Bloemsaat JG, Meulenbroek RGJ, Van Galen GP. Differential effects of mental load on proximal and distal arm muscle activity. Exp Brain Res. 2005;167(4):622-634.

[11] Zadry HR, Dawal SZ, Taha Z. The relation between upper limb muscle and brain activity in two precision levels of repetitive light tasks. Int J Occup Saf Ergon. 2011;17(4): 373-384. doi:10.1080/10803548.2011.11076901.

[12] Zadry HR, Dawal SZ, Taha Z. Combination of electromyography and electroencephalography measurements in designing repetitive task in industry. Adv Sci Lett. 2011;4: 2498-2502.

[13] Karwowski W, Marras WS. Occupational ergonomics: engineering and administrative controls. Boca Raton (FL): CRC Press; 2003.

[14] Szeto GPY, Lin JKM. A study of forearm muscle activity and wrist kinematics in symptomatic office workers performing mouse-clicking tasks with different precision and speed demands. J Electromyogr Kinesiol. 2011;21:59-66.

[15] Nakata M, Hagner IM, Jonsson B. Perceived musculoskeletal discomfort and electromyography during repetitive light work. J Electromyogr Kinesiol. 1992;2(2):103-111.

[16] Escorpizo RS, Moore AE. Quantifying precision and speed effects on muscle loading and rest in an occupational hand transfer task. Int J Ind Ergon. 2007;37(1):13-20.

[17] Wartenberg C, Dukic T, Falck A, et al. The effect of assembly tolerance on performance of a tape application task: a pilot study. Int J Ind Ergon. 2004;33:369-379.

[18] Li G, Buckle P. A practical method for the assessment of work-related musculoskeletal risks quick exposure check (QEC). Proc Hum Fact Ergon Soc Annu Meet. 1998;42(19):1351-1355. 
[19] Konrad P. The ABC of EMG. A practical introduction to kinesiological electromyography. Scottsdale (AZ), Noraxon; 2005.

[20] Sommerich C, Joines S, Hermans V, et al. Use of surface electromyography to estimate neck muscle activity. J Electromyogr Kinesiol. 2000;10(6):377-398.

[21] Minning S, Eliot CA, Uhl TL, et al. EMG analysis of shoulder muscle fatigue during resisted isometric shoulder elevation. J Electromyogr Kinesiol. 2007;17(2):153-159.

[22] Forsyth A, Hiler R, Michels J, et al. Electromyography (EMG) of the trapezius muscles during clerical work. J Under Res. 2000;3:6:287-292.

[23] Hermens HJ, Freriks B, Disselhorst-Klug C, et al. Development of recommendations for SEMG sensors and sensor placement procedures. J Electromyogr Kinesiol. 2000;10(5):361-374.

[24] Andreassi J. Psychophysiology: human behavior and physiological response. Mahvah (NJ): Erlbaum; 2007.

[25] Teplan M. Fundamentals of EEG measurement. Meas Sci Rev. 2002;2(2):1-11.

[26] Bosch T, De Looze M, Kingma I, et al. Electromyographical manifestations of muscle fatigue during different levels of simulated light manual assembly work. J Electromyogr Kinesiol. 2009;19(4):e246-e256.

[27] Bartuzi P, Roman-Liu D, Wiśniewski T. The Influence of fatigue on muscle temperature. Int $\mathrm{J}$ Occup Saf Ergon. 2012;18(2):233-243. doi:10.1080/10803548.2012. 11076931.

[28] Taelman J, Van Huffel S, Spaepen A. Wavelet-independent component analysis to remove electrocardiography contamination in surface electromyography. IEEE Eng Med Biol. 2007;1:682-5.

[29] Basmajian J, DeLuca CJ. Muscles alive: their functions revealed by electromyography. 5th ed. Baltimore (MD): Williams and Wilkins; 1985.

[30] Gerleman DG, Cook TM. Instrumentation. In: Soderberg GL, editor. Selected topics in surface electromyography for use in the occupational setting: expert perspectives. US Department of Health and Human Services - National Institute for Occupational Safety and Health; 1992. p. 4368. Available from: http://www.cdc.gov/niosh/docs/91-100/ pdfs/91-100.pdf

[31] De Luca G. Fundamental concepts in EMG signal acquisition. Delsys; 2003. Available from: https://www.delsys. com/Attachments_pdf/WP_Sampling1-4.pdf.

[32] Portney LG, Watkins MP. Foundations of clinical research. Upper Saddle River (NJ): Pearson/Prentice Hall; 2000.

[33] Lane, David M. Standard error of the estimate. [cited 2014 March 12]. Available from: http://onlinestatbook.com/2/ regression/accuracy.html

[34] Zhang FR, He LH, Wu SS, et al. Quantify work load and muscle functional activation patterns in neckshoulder muscles of female sewing machine operators using surface electromyogram. Chin Med J. 2011;124(22): $3731-3737$.

[35] Julie N, Côté JN, Raymond D, et al. Differences in multijoint kinematic patterns of repetitive hammering in healthy, fatigued and shoulder-injured individuals. Clin Biomech. 2005;20(6):581-590.

[36] Andersen J, Kaergaard A, Mikkelsen S, et al. Risk factors in the onset of neck/shoulder pain in a prospective study of workers in industrial and service companies. Occup Environ Med. 2003;60(9):649-654.

[37] Nussbaum MA. Static and dynamic myoelectric measures of shoulder muscle fatigue during intermittent dynamic exertions of low to moderate intensity. Eur J Appl Physiol. 2001;85(3):299-309.
[38] Tetteh EG. A pilot laboratory study of EMG back activity among normal versus overweight workers during material handling on multi-level racks [doctoral dissertation]. West Lafayette (IN): Purdue University; 2007.

[39] Farina D, Arendt-Nielsen L, Merletti R, et al. Effect of experimental muscle pain on motor unit firing rate and conduction velocity. J Neurophysiol. 2004;91(3):12501259.

[40] Hägg G. Static workload and occupational myalgia - a new explanation model. In: Andersen PA, Hobart DJ, Danoff JV, eds. Electromyographical Kinesiology. Amsterdam: Elsevier; 1991. p. 141-144.

[41] Waersted M. Human muscle activity related to nonbiomechanical factors in the workplace. Eur J Appl Physiol. 2000;83(2):151-158.

[42] Pal N, Chuang C, Ko L, et al. EEG-based subject-and session-independent drowsiness detection: an unsupervised approach. EURASIP J Adv Sig Pr. 2008;2008:1-11.

[43] Seen K, Mohd Tamrin S, Meng G. Driving fatigue and performance among occupational drivers in simulated prolonged driving. Glob J Health Sci. 2010;2(1): 167-177.

[44] Åkerstedt T, Kecklund G, Gillberg M. Sleep and sleepiness in relation to stress and displaced work hours. Physiol Behav. 2007;92(1-2):250-255.

[45] Jap BT, Lal S, Fischer P, et al. Using EEG spectral components to assess algorithms for detecting fatigue. Expert Syst Appl. 2009;36 (2 Pt 1), 2352-2359.

[46] Casey A, Benson H, O'Neill BE. The Harvard medical school guide to lowering your blood pressure. New York (NY): McGraw-Hill; 2005.

[47] Abdul-latif AA, Cosic I, Kumar DK, et al. Power changes of EEG signals associated with muscle fatigue: the root mean square analysis of EEG bands. In: Proceedings of the Intelligent Sensors, Sensor Networks and Information Processing Conference, Australia, 2004. p. 534-534.

[48] Au AK, Keir PJ. Interfering effects of multitasking on muscle activity in the upper extremity. J Electromyogr Kinesiol. 2007;17(5):578-586.

[49] Iwanaga K, Saito S, Shimomura Y, et al. The effect of mental loads on muscle tension, blood pressure and blink rate. J Physiol Anthropol Appl Human Sci. 2000;19(3): $135-141$

[50] Krantz G, Forsman M, Lundberg U. Consistency in physiological stress responses and electromyographic activity during induced stress exposure in women and men. Integr Phys Beh Sci. 2004;39(2):105-118.

[51] Lundberg U, Forsman M, Zachau G, et al. Effects of experimentally induced mental and physical stress on motor unit recruitment in the trapezius muscle. Work Stress. 2002;16(2):166-178.

[52] Lundberg U. Psychophysiology of work: stress, gender, endocrine response, and work-related upper extremity disorders. Am J Ind Med. 2002;41(5):383-392.

[53] Coorevits PLM, Danneels LA, Ramon H, et al. Statistical modelling of fatigue-related electromyographic median frequency characteristics of back and hip muscles during a standardized isometric back extension test. J Electromyogr Kinesiol. 2005;15:444-451.

[54] Solnik S, Devita P, Grzegorczyk K, et al. EMG frequency during isometric, submaximal activity: a statistical model for biceps Brachii. Acta Bioeng Biomech. 2010;2(3): 21-8.

[55] Finsen L, Sogaard K, Jensen C, et al. Muscle activity and cardiovascular response during computer mouse work with and without memory demands. Ergonomics. 2001;44(14):1312-1329. 
[56] Choobineh A, Tabatabaei SH, Mokhtarzadeh A, et al. Musculoskeletal problems among workers of an Iranian rubber factory. J Occup Health. 2007;49(5):418-423.

[57] Coury HJCG, Porcatti IA, Alem MER, et al. Influence of gender on work-related musculoskeletal disorders in repetitive tasks. Int J Ind Ergon. 2002;29(1):33-39.
[58] Rangan S, Van Dongen HPA. Quantifying fatigue risk in model-based fatigue risk management. Aviat Space Environ Med. 2013;84:155-157.

[59] Byström SEG, Mathiassen SE, Fransson-Hall C. Physiological effects of micropauses in isometric hand grip exercises. Eur J Appl Physiol. 1991;63:405-411. 